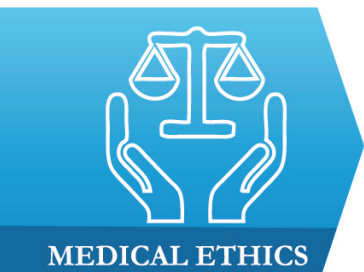

Faculty of Medicine, Transilvania University of Brasov, Romania
DOI: $10.15386 / \mathrm{mpr}-1223$

Manuscript received: 29.10 .2018

Received in revised form: 23.05.2019

Accepted: 13.07.2019

Address for correspondence:

nadinneroman@gmail.com

\section{Ethical considerations about informed consent in physiotherapy in Romania}

Nadinne Roman, Roxana Miclaus, Liliana Rogozea

\begin{abstract}
Background and aim. Physiotherapy has a distinct feature in terms of patient relationship, regarding the level of communication, especially how physical therapy sessions take place throughout the rehabilitation process. One of the particularities encountered in Romanian physiotherapy practice is related to obtaining informed consent (IC), considering that the initial contact of the patient is with a rehabilitation doctor when general consent is obtained. The aim of this study was to investigate Romanian physiotherapists aspects related to the frequency and use of IC at physiotherapy onset.

Method. A cross-sectional study was conducted among Romanian physiotherapists using a survey with 2 open and 37 closed items, including nine demographics items, developed after a systematic literature review. The closed items were Likert type scales, measuring frequency or agreement on scale from 1 to 5 . The questionnaire was distributed online from November 2017 until May 2018 and data was gathered from 148 physiotherapists. The statistical analysis targeted descriptive analysis and multiple regression.
\end{abstract}

Results. The results suggested that only $40.82 \%$ of physiotherapists are always seeking to obtain IC at physiotherapy onset, while $16.3 \%$ never apply this legal demand in their professional activity, confidence intervals were set to $95 \%$ with 3.34 lower bound and 3.82 upper bound. Through multiple regression, we have identified factors which correlates with an increased frequency of IC obtaining at physiotherapy onset. Professional skills, ethical knowledge and ethical reasoning are factors with a positive influence towards obtaining IC with an increased frequency.

Conclusions. Ethical knowledge, moral reasoning and consideration for patient are elements which have a positive influence regarding the awareness of IC. The process of demanding and obtaining IC within Romanian physiotherapists must be improved despite the particularities of medical practice. Further research is needed to identify the methods by which Romanian physiotherapists awareness toward IC can be increased.

Keywords: physiotherapy, ethics, informed consent, knowledge

\section{Background and aims}

Physiotherapy as a medical field has been expanding and developing over the last decades, becoming an important component of the medical system. Physiotherapists have become responsible for the rehabilitation of patients in a wide range of conditions, correlated with all medical specialties, evolving and also becoming a distinct component of emergency medicine [1]. Physiotherapy ethical challenges have accompanied the field development, but the bioethical questions have not been sufficiently assimilated to academic studies [2]. The medical evolution during the last century, both in terms of medical practice and technology evolution, demanded the development of coherent moral and ethical policies, objective and well-defined, needed to intervene in protecting the patient and human life [5]. One of these tools is represented by informed consent. The informed consent, in addition to protecting human subjects included in the medical research, is used to respect and promote the patients' autonomy and to protect them 
from mischief or harmful actions to his or her health $[3,4]$.

Multiple methods have been tried to make obtaining informed consent more efficient over the years of medical practice, but most have proven to be difficult, long or twisted, by the use of complex words for people who are not familiar with medical terms, and whose effectiveness has not been proven so far.

The specificity of physiotherapy session influences the IC process and physiotherapists face ethical challenges within this context. During physical therapy sessions, the physiotherapist has to work progressively with the patient, in order to change techniques and methods of treatment, the type of exercises. Considering that physiotherapy is a medical treatment method, is it necessary to re-use the IC process when changing the techniques used? Here is one of the problems of informed consent in physiotherapy: how should it be achieved and how much does interfere with the quality of the medical act? [6,7] There is no framework or guidelines about how informed consent should be obtained in physiotherapy, neither in practice or research. Although gathering and demanding IC is a legally demand, sometimes it is poorly understood and applied [8]. While in some cases, physiotherapists use different types of consent like: document consent, verbal consent and general consent usually taken at the beginning of physiotherapy, and also the so called "body language consent", before and during treatment to see the patients' reaction, their compliance during treatment and the presence of a possible objection to treatment [9].

The physiotherapist profession in Romania has developed through the last century, but until 2016, when the profession begun the regulation process, no legislation was specific for Romanian physiotherapists. Within the context of professional development, legislation and ethical approach of physiotherapy profession, issues related to IC have not been approached before [10]. The aim of our study was to investigate Romanian physiotherapists aspects concerning the process of acquiring and obtaining IC.

\section{Methods}

A questionnaire was developed after a systematic literature review and a cross-sectional study was made. The review was made upon ISI Web of Science Core Collection interrogation, using specific keywords related to "physiotherapy", "ethics", "dilemmas", “informed consent". For the final analysis 24 articles were revised. After extracting the main ethical issues from the selected papers, a questionnaire was developed based on the existing issues found in the literature. A face validity was assured by two bioethics teachers, interfering with the ethical aspects of the questionnaire formulation. After this phase, the questionnaire was discussed and tested with seven physiotherapists regarding addressability, comprehension and practice utility. Survey Monkey platform was used to assess IC obtaining among the Romanian Physiotherapists.
The survey consisted of 2 open and 37 closed items, including nine demographic items. The majority of closed items were Likert type scales, measuring frequency or agreement on scale from 1 to 5 (as 1 considered to "Never" or "At all" and 5 considered as "Always" or "Very Important"). Ethical approval (no. 6/2017) was granted from the Ethical Committee of Transilvania University of Brasov, as part of a doctoral thesis research. The survey was disseminated online, using email, through professional associations and professional communities on social networks, from November 2017 until May 2018. In Romania, the physiotherapists national professional association is at the stage of development and organization, so no information exists regarding the number of physiotherapists who practice. A total of 148 responses were collected and analyzed through the SPSS (The Statistical Package for the Social Sciences) software. The statistical analysis targeted Cronbach's Alpha value, factorial analysis for internal validation, descriptive analysis and multiple regression.

The questionnaire analysis of reliability was calculated using Cronbach's Alpha coefficient which indicates a minimum $\alpha$ coefficient between 0.65 and 0.8 [11].

For internal validity a factor analysis was accomplished, with Equamax rotation Kaiser normalization and using Kaiser-Meyer-Olkin Measure of Sampling Adequacy and Bartlett's test of Sphericity values to determine the use of factor analysis for reducing the number of factors. Factors with eigenvalue greater than 1 were extracted.

In our research, one of the main purposes was to figure out the physiotherapist's professional behavior which might influence the frequency of IC obtaining and the reasons behind, except legal demanding. A multiple regression was applied using the statistical software, to predict elements which influence a high frequency regarding IC obtaining at physiotherapy onset. Linearity was assessed by investigating partial regression plots. Durbin-Watson value of 1.961 assured the independence of residuals. The assumption of normality was met, as assessed by a Q-Q Plot inspection. Confidence intervals (CI) for data analysed was set to $95 \%$. Multiple regression is an extension of linear regression. It is used when predicting or associating the value of a variable based on the value of two or more other variables. In our analysis we considered as a dependent variable the frequency of IC demanding at the onset of physiotherapy and we used the other items of the questionnaire as independent variables.

A raised $\mathrm{R}^{2}$ adjusted values, from 0.3 to 0.5 suggest a medium effect size, while values greater than 0.5 suggest a large effect size [12]. $\mathrm{R}^{2}$ values are associated by the $\mathrm{p}$-values,significant when below 0.05 . To establish the variables which might predict frequency of IC obtaining when multiple regression was applied, stepwise method was chosen, the reason why the last predictor includes all anterior predictors, in a descending order. 


\section{Results}

The questionnaire analysis of reliability was calculated using Cronbach's Alpha coefficient and the value of 0.851 indicated a good reliability of the data. For internal validity a factor analysis was accomplished, with Equamax rotation and Kaiser normalization. KaiserMeyer-Olkin Measure of Sampling Adequacy value of 0.721 and Bartlett's test of Sphericity, with $\mathrm{X}^{2}=$ 4903.155 allowed us to use factor analysis for reducing the number of factors. Factors with Eigenvalue greater than 1 were extracted, resulting a total of 9 factors. The factors were associated with: Professional development (1), Professional issues (2), Ethical issues and knowledge (3), Time management (4), Competences (5), Team work (6), Informed consent (7), Patient Autonomy (8) and Patient-Physiotherapist Relationship (9). A total number of 148 physiotherapists completed the survey. The participants gender distribution was $68.92 \%$ females and $31.08 \%$ males. The age median was 31.59 years, within a range from 21 to 65 years. A percent of $29.06 \%$ of physiotherapists working in public sector, 65.55 in private practice and $5.39 \%$ in other structures like physiotherapy resorts. Regarding the studies degree, $5.39 \%$ were college graduates, $60.14 \%$ with university studies, $33.11 \%$ with master degree and $1.36 \%$ (2 respondents) with $\mathrm{PhD}$ degree.

\section{Descriptive analysis}

The item addressed to physiotherapists questioned them regarding the frequency of IC obtaining before physiotherapy program proceeding. Only $40.82 \%$ of physiotherapists were always seeking to obtain IC from their patients as the therapy begin. With an average frequency, $19.73 \%$ of Romanian physiotherapists request and obtain IC from their patients, $12.9 \%$ apply it from time to time, $10.2 \%$ declared they were rarely requesting IC and $16.3 \%$ (24 from 148) never apply this legal demand in their professional activity, with CI $95 \%$ and lower bound 3.34 and upper bound 3.82 .

The frequency of IC consent obtaining in physiotherapy, when Romanian physiotherapists are changing the techniques or the type of interventions (the type of therapeutic exercises) are presented in Table I. The results prove that the percent of physiotherapists seeking to obtain IC within this case is even lower compared to physiotherapy onset IC obtaining.

Multiple linear regression

The first predictor, as shown in table II is related to the frequency of IC reuse when therapy plan is modified a, it has a statistically significant predictability due to $\mathrm{F}$ changed $\mathrm{p}<0.005$ and by $\mathrm{R}$ value of 0.694 indicates a strong level of association, with adjusted $\mathrm{R}^{2}$ of $47.1 \%$ and a medium effect size.

Table I. Frequency expressed in percentages of IC obtaining in physiotherapy, when physiotherapists are changing the techniques.

\begin{tabular}{|c|c|c|c|c|c|c|}
\hline Never & Occasionally & Average & Frequent & Always & Mean & 95\% CI for Mean \\
\hline 21.77 & 16.33 & 10.88 & 20.41 & 30.61 & 3.58 & $\begin{array}{l}\text { Lower Bound } \\
\text { Upper Bound }\end{array}$ \\
\hline
\end{tabular}

Table II. Multiple regression regarding elements which influence IC demanding at physiotherapy onset.

\begin{tabular}{|c|c|c|c|c|c|c|c|c|c|}
\hline \multicolumn{10}{|c|}{ Model Summary } \\
\hline \multirow{2}{*}{ Model } & \multirow{2}{*}{$\mathbf{R}$} & \multirow{2}{*}{$\mathbf{R}^{2}$} & \multirow{2}{*}{ Adjusted R ${ }^{2}$} & \multirow{2}{*}{$\begin{array}{l}\text { Std. Error of } \\
\text { the Estimate }\end{array}$} & \multicolumn{5}{|c|}{ Change Statistics } \\
\hline & & & & & $\mathbf{R}^{2}$ Change & F Change & df1 & df2 & Sig. F Change \\
\hline 1 & $.694^{\mathrm{a}}$ & .481 & .476 & 1.015 & .481 & 92.827 & 1 & 100 & 0.000 \\
\hline 2 & $.749^{\mathrm{b}}$ & .561 & .552 & .939 & .080 & 17.958 & 1 & 99 & 0.000 \\
\hline 3 & $.774^{\mathrm{c}}$ & .599 & .587 & .902 & .038 & 9.252 & 1 & 98 & 0.003 \\
\hline 4 & $.785^{\mathrm{d}}$ & .617 & .601 & .886 & .018 & 4.524 & 1 & 97 & 0.036 \\
\hline 5 & $.799^{\mathrm{e}}$ & .639 & .620 & .864 & .022 & 5.967 & 1 & 96 & 0.016 \\
\hline 6 & $.809^{\mathrm{f}}$ & .655 & .633 & .850 & .016 & 4.299 & 1 & 95 & 0.041 \\
\hline 7 & $.820^{\mathrm{g}}$ & .673 & .648 & .832 & .018 & 5.174 & 1 & 94 & 0.025 \\
\hline 8 & $.830^{\mathrm{h}}$ & .689 & .662 & .815 & .016 & 4.880 & 1 & 93 & 0.030 \\
\hline 9 & $.838^{\mathrm{i}}$ & .702 & .673 & .802 & .013 & 4.126 & 1 & 92 & 0.045 \\
\hline 10 & $.845^{\mathrm{j}}$ & .715 & .684 & .789 & .012 & 3.953 & 1 & 91 & 0.048 \\
\hline
\end{tabular}

a, Written IC request ${ }^{b}$, Rehabilitation protocols needed ${ }^{\mathrm{c}}$, Patient assessment during physiotherapy ${ }^{\mathrm{d}}$, Ethics Knowledge ${ }^{\mathrm{e}}$, Monthly professional discussions ${ }^{\mathrm{f}}$, Ethical Reasoning ${ }^{\mathrm{g}}$, Communication competencies ${ }^{\mathrm{h}}$, Professional Courses at workplace ${ }^{\mathrm{i}}$, Consideration for Patient. ${ }^{\mathrm{j}}$. Predictors: (Constant), IC reobtaining at therapy modify. ${ }^{\mathrm{k}}$, Dependent Variable: IC demanding at physiotherapy onset. 
The second predictor is composed by the first predictor and the variable related to obtaining CI in written form $^{\mathrm{b}}$, it has a statistically significant predictability due to $\mathrm{F}$ changed $p<0.005$ and by $R$ value of 0.749 indicates a strong level of association, with adjusted $\mathrm{R}^{2}$ of $55.2 \%$ and a large effect size. But the most appropriate and broad predictor or model is the last one [10], consisting of ten variables as shown in Table II ${ }^{\mathrm{j}}$. The $\mathrm{R}$ value of 0.845 for the tenth model, indicate a very strong linear relationship between the predictor and the independent variable (frequency of IC obtaining at physiotherapy onset ${ }^{k}$ ). The adjusted $\mathrm{R}^{2}$ value of $68.4 \%$ denote a large effect size and according to $F$ changed threshold of statistical significance, of 0.048 , the model fits and is suitable for explaining the predictability for the independent variable.

\section{Discussion}

Since the first predictor which contains a single variable is related to the process of IC reuse when the physical therapy is modified, the outcome suggests that the physiotherapists who tend to re-take the IC process when there are changes in the therapy, also demand IC at physiotherapy onset. Actions and behaviors related to other nine variables: Rehabilitation protocols needed ${ }^{\mathrm{c}}$, Patient assessment during physiotherapy d, Ethics Knowledge ${ }^{\mathrm{e}}$, Monthly professional discussions ${ }^{\mathrm{f}}$, Ethical Reasoning ${ }^{\mathrm{g}}$, Communication competencies ${ }^{\mathrm{h}}$, Continuous Professional Courses at workplace ${ }^{i}$, Consideration for Patient ${ }^{j}$ are factors which influence in a positive manner the process of IC demanding and obtaining when physiotherapy begin (Table II).

Physiotherapists who have been reported at an increased frequency of IC obtaining at the onset of physiotherapy, have also been reported at a high level of ethical knowledge, have had a better moral reasoning for decision-making in the case of an ethical problem and presented a high level of interest in consideration for the patient. It seems that an increased professional awareness related to Continuous Professional Development (especially at workplace), professional team discussions, recognition of the need for the medical protocols' implementation, patient's assessment and an adequate level of communication competencies also influence the awareness regarding the process of IC demanding among the Romanian physiotherapists.

Swisher in a retrospective analysis focused on ethical issues in physiotherapy, classified the stages of the evolution of the physiotherapist's profession, granted the period 1980-1989 credit for the application of the informed consent. Considering the results of our research, found in descriptive analysis, it seems that Romanian physiotherapists have not sufficiently assimilated ethical concepts regarding IC in medical practice [13]. The evolution of medicine and also of physiotherapy practice rise new responsibilities and demands high standards of practice [14]. And the results from our research suggest a need for improving ethical dimensions of physiotherapy practice.

Although in Romania, the physiotherapists are working under a physician's prescription and supervision, the physical therapy program is a medical act implemented by the physiotherapist. The physiotherapists' skills are to assess and implement physical therapy programs, based on functional evaluation $[15,16]$. Scientific literature deals with issues related to IC from physiotherapists perspective due to increased responsibilities and the lack of a specific framework toward how IC should be obtained in physiotherapy [17]. But since the physical therapy plan is a medical action, the patient must be involved within the medical decision regarding physical exercises [18]. Considering that only $40.82 \%$ from 148 Romanian physiotherapists are always obtaining IC from the patients at the onset of treatment, suggest there is a need for informational and educational tools to raise physiotherapists' awareness of this important component of medical practice.

During physical therapy sessions (medical gymnastics), there is a dynamic relation between the physiotherapist and the patient: the physiotherapist works progressively with the patient, s/he changes techniques and replaces methods of treatment. One of the major problems in physiotherapy, is the lack of certainty as of how to obtain a proper IC $[19,20]$. Given the fact that the therapeutic techniques used during a physiotherapy session may change, it is necessary to obtain an IC is for each new applied method, or not? The lack of a framework in this concern is approached by bioethicists and remain as an unsolved issue. The low frequency of IC reuse in Romanian physiotherapy practice, as results from Table I, when the physical therapy dynamics change is reflecting anterior research regarding the lack of a concrete framework [2,21-25].

Ethical literature in the field of physiotherapy has approached very little of the ethical issues faced by physiotherapists in medical practice. Analyzing and exposing the factors that influence the demand and obtaining IC in physiotherapy offers a wider framework for understanding the shortcomings or the needs of physiotherapists from this point of view. The correlation regarding factors related to ethical knowledge, ethical reasoning and professional development with a high frequency of IC demanding and awareness of this process, provides important information for a better understanding regarding IC in physiotherapy (Table II). As previous research has shown that physiotherapists profession evolution demands new ethical training, our research proves the importance of ethical knowledge in IC process related to physiotherapy services [26]. 
The elements which influence physiotherapists perceptions and behaviors toward IC demanding are factors which must be investigated and analyzed, due to the complexity of IC process, related to communication, legislation and ethical components[27-29]. A recent debate article suggest that physiotherapists should provide useful information to the patient related to patient's needs and understandings, so the patient can make a "good "decision after information provided. But related to our research results, the first need is to request and obtain IC, as a physiotherapist, but since $16.3 \%$ of 148 physiotherapists never request IC at physiotherapy onset and $10.2 \%$ are applying this request rarely, health management and legally aspects must be implemented first.

It remains unclear how IC process can be improved among Romanian physiotherapists and further research is needed to establish how and if this process can be improved through professional development and educational tools.

The limits of the research are represented by the small sample size of participants in the study and the fact that there is no national evidence in Romania regarding the total number of practicing physiotherapists.

\section{Conclusions}

Demanding and obtaining IC from the perspective of physiotherapists on the territory of Romania is a process that requires improvement, given the fact that it is a legal right of patients and must be respected, with or without prior general consent obtaining from physician. The percentage of physiotherapists who always requires and obtain IC at the onset of treatment rise ethical and legal issues of practice and impact the quality of physiotherapy services. Factors which influence this negative aspect of the physiotherapist profession must be further investigated.

Ethical knowledges and professional development related to team discussion, workplace courses, communication competencies and moral reasoning were associated with a higher frequency in IC demanding at physiotherapy onset.

\section{References}

1. Farrell SF. Can physiotherapists contribute to care in the emergency department? Australas Med J. 2014;7:315-317.

2. Poulis I. Bioethics and physiotherapy. J Med Ethics. 2007;33:435-436.

3. Parsons T. Essays in Sociological Theory. Revised edition ed. Press TF, editor.; 1954: pp. 34-50.

4. Tam NT, Huy NT, Thoa le TB, Long NP, Trang NT, Hirayama $\mathrm{K}$, et al. Participants' understanding of informed consent in clinical trials over three decades: systematic review and metaanalysis. Bull World Health Organ. 2015;93:186H-198H.
5. Albala I, Doyle M, Appelbaum PS. The evolution of consent forms for research: a quarter century of changes. IRB. 2010;32:7-11.

6. Legea nr. 229/2016 privind organizarea și exercitarea profesiei de fizioterapeut, precum și pentru înființarea, organizarea și funcționarea Colegiului Fizioterapeuților din România [Law no. 229/2016 regarding the organization and practice of physiotherapy, as well as founding, organization and function of the Romanian College of Physiotherapists]. Monitorul Oficial nr. 939 din 22 noiembrie 2016. Available from: http:// www.monitoruljuridic.ro/act/lege-nr-229-din-17-noiembrie2016-privind-organizarea-i-exercitarea-profesiei-defizioterapeut-precum-i-pentru-nfiin-area-organizarea-i-funcionarea-colegiului-fizioterapeu-ilor-din-rom-nia-183839. $\mathrm{html}$

7. JaegerL, Bertram E, Grate S, Mischkowsky T, PaulD, Probst J, et al.Patients' rights--doctors' duties. Versicherungsmedizin. 2015;67:70-74.

8. Delany CM. Respecting patient autonomy and obtaining their informed consent: ethical theory-missing in action. Physiotherapy. 2005;91:197-203.

9. Fenety A, Harman K, Hoens A, Bassett R. Informed consent practices of physiotherapists in the treatment of low back pain. Man Ther. 2009;14:654-660.

10. Hudon A, Drolet MJ, Williams-Jones B. Ethical issues raised by private practice physiotherapy are more diverse than first meets the eye: recommendations from a literature review. Physiother Can. 2015;67:124-132.

11. Bland JM, Altman DG. Cronbach's alpha. BMJ. 1997;314:572.

12. Cohen J, Cohen P, West SG, Aiken LS. Applied multiple regression/correlation analysis for the behavioral sciences. 3rd ed. Mahwah, NJ: Lawrence Erlbaum Associates, 2003: pp. 703.

13. Swisher LL.A retrospective analysis of ethics knowledge in physical therapy (1970-2000). Phys Ther. 2002;82:692-706.

14. Gibson BE, Nixon SA, Nicholls DA. Critical reflections on the physiotherapy profession in Canada. Physiother Can. 2010;622:98-100, 101-103.

15. WCPT. World Confederation of Physical Therapy. What is physical therapy. Oct. 2016 Available from: https://www. wcpt.org/what-is-physical-therapy.

16. ILO. Updating the International Standard Classification of Occupations (ISCO). Available from: http://www.ilo.org/ public/english/bureau/stat/isco/docs/health.pdf

17. Elkin S. Informed consent: requirements for legal and ethical practice. Physiotherapy Theory and Practice - Physiother Theory Pract. 2001;17:97-105.

18. Entwistle VA, Watt IS. Patient involvement in treatment decision-making: the case for a broader conceptual framework. Patient Educ Couns. 2006;63:268-278.

19. Delany CM. In private practice, informed consent is interpreted as providing explanations rather than offering choices: a qualitative study. Aust J Physiother. 2007;53:171-177.

20. Delany C, Galvin J. Ethics and shared decision-making in paediatric occupational therapy practice. Dev Neurorehabil. 2014; 17:347-354. 
21. Cassileth BR, Zupkis RV, Sutton-Smith K, March V. Informed consent -- why are its goals imperfectly realized? N Engl J Med. 1980;302:896-900.

22. Copnell G. Informed consent in physiotherapy practice: it is not what is said but how it is said. Physiotherapy. 2018;104:67-71.

23. Hall DE, Prochazka AV, Fink AS. Informed consent for clinical treatment. CMAJ. 2012;184:533-540.

24. Purcaru D, Preda A, Popa D, Moga MA, Rogozea L. Informed consent: how much awareness is there? PLoS One. 2014;9:e110139.

25. Rogozea L, Diaconescu DE, Dinu EA, et al. Biomedical research ethics. Rom J Morphol Embryol. 2014;55(Suppl 2):719-722.

26. Hudon A, Laliberté M, Hunt M, Sonier V, Williams-Jones
B, Mazer B, et al. What place for ethics? An overview of ethics teaching in occupational therapy and physiotherapy programs in Canada. Disabil Rehabil. 2014;36:775-780.

27. Schenker Y. Fernandez A, Sudore R,Schillinger D. Interventions to improve patient comprehension in informed consent for medical and surgical procedures a systematic review. Med Decis Making. 2010;31:151-173.

28. Rogozea L, Miclaus R, Nemet C, Balescu A, Moleavin I. Education, ethics and e-Communication in medicine. Proceeding DIWEB'08 Proceedings of the 8th WSEAS international conference on Distance learning and web engineering. 2008: pp. 197-201.

29. Dima L, Repanovici A, Purcaru D, Rogozea L. Informed consent and e-communication in medicine. Revista Romana de Bioetica. 2014;12:37-46. 